\title{
Using as-synthesized mesoporous silica as new capturer and releaser
}

\section{Mini review}

A new concept is lodged and realized in this mini review, that is, how to exploit the template micelles occluded in the channel of assynthesized mesoporous silica materials. Organic template, ionic or non-ionic surfactants or amphiphilic block copolymers, is necessary for inducing the wall structure of mesoporous material through nanocasting or structure -directing procedure. During the synthesis of mesoporous molecular sieves, the assembling and condensation of inorganic materials are performed around the template micelles, forming the MCM-41 over cetyl trimethyl ammonium bromide (CTAB) or SBA-15 on P123 micelles. ${ }^{1,2}$ However, after the synthesis of mesoporous silica, these templates usually need to be removed from these molecular sieves by calcinations at high temperature or extraction with alcohol in order to empty the channel of the mesoporous materials, which costs a lot of energy and time and sometimes it will cause waste and pollutant. Moreover, the template removal step unavoidably leads to the consumption of silanol groups and the structure shrinkage, harmful for the potential application of these materials such as adsorption and catalysis.

In fact, these template micelles have constant chemical composition and relative regular geometric structure. For example these micelles occluded in the as-synthesized mesoporous silica such as MCM-41 are the unique resource, since they are distributed in the spoke-like configuration to form numerous sub-nanometer gaps between them and the silica walls, and these inherent chemical and geometric properties are very difficult, if not impossible, to be duplicated by other methods. ${ }^{3}$ For this reason, it is necessary to utilize these template micelles orientated inside the channel. We had reported a simple approach previously: the as-made ordered mesoporous materials with template occluded in the pores were utilized as the specific support and manually ground with guest precursor salts such as magnesium acetate. And this mixture was then calcined to form high dispersion of guest species like $\mathrm{MgO}$, performing the guest dispersion and the template removal at one step. ${ }^{4}$ As the consequence, a solventfree technique was employed for fast modification of mesoporous materials. Copper, chromium and iron oxide species could be highly dispersed in SBA-15 by manually grinding the corresponding precursor salts and the host, followed by calcinations for the first time. This method is more effective to spontaneously disperse oxide species onto SBA-15 than impregnation. Besides, $\mathrm{Cr}(\mathrm{VI})$ species dominate in the mixing sample while $\mathrm{Cr}$ (III) species dominate in the impregnation one. In the temperature programmed surface reaction of nitrosamines, the sample prepared by solvent-free method showed a higher catalytic activity than the impregnation one. ${ }^{5}$ This method finely exploits the weak interaction of inorganic-organic hybrids to achieve spontaneous infiltration of guest species in the occluded pores of the as-made mesoporous materials so as to be a powerful means to fabricate guesthost composites. More importantly, this method hints a possibility to take advantage of the confined space between silica wall and template aggregates. ${ }^{4,6}$

On the other hand, we exploited and utilized these template

\author{
Volume 2 Issue I - 2018
}

\author{
Jie Ling Chen,',2 Mi Mi Wan,' Xin Yu Ming \\ Dong, 'Ying Wang, ' Jian Hua Zhu' \\ 'Key Laboratory of Mesoscopic Chemistry of MOE, College of \\ Chemistry and Chemical Engineering, Nanjing University, China \\ ${ }^{2}$ College of Chemistry and Chemical Engineering, Nanjing \\ University, China
}

\begin{abstract}
Correspondence: Jian Hua Zhu, Key Laboratory of Mesoscopic Chemistry of MOE, College of Chemistry and Chemical Engineering, Nanjing University, China, Email jhzhu@nju.edu.cn Ying Wang, College of Chemistry and Chemical Engineering, Nanjing University, China, Email wangy@nju.edu.cn
\end{abstract}

Received: December 20, 2017 | Published: February 20, 2018

micelles occluded in the channel of mesoporous materials to compose new functional materials or adjust the mean pore sizes of the porous solid. The main channel diameters of mesoporous silica or mesoporous other metal oxides is fixed, it is true, and this property is of great importance for some cases of catalyst or adsorbents. However, in some complex situations where the hierarchical structure is needed for capturing the target molecules having different molecular diameters and conformations, say, to trap the carcinogenic nitrosamines in cigarette smoke in which small volatile nitrosamines and the bulky tobacco specific nitrosamines exist, the consumers hope to have new mesoporous composite in which some guests form something like cobweb to hold up or capture the nitrosamines with different shape and sizes. ${ }^{7}$ On the other hand, although appearance of order mesoporous materials affords a new type of candidate for catalysts and adsorbents, because their large pore size enables the active centers accessible for the large feedstock molecules. However, it is still difficult for them to replace the common microporous sorbents based on zeolite, and one of the reasons is their inherent weakness. For example, either MCM41 or SBA-15 consist of only silica and suffer the lack of metal ion so that they lack the necessary active species for adsorption of small target like $\mathrm{CO}_{2}{ }^{3,8}$ For this reason, we decided to exploit the template occlude the channel of SBA-15 as the soft supports to disperse amine, creating new $\mathrm{CO}_{2}$ capturer. This new strategy not only save the energy and time as well as reduce the production of pollutant formed during the removal of template micelles, but also enhance the efficiency of encapsulated amine for $\mathrm{CO}_{2}$ adsorption because the existence of $\mathrm{P} 123$ micelles improves the accessibility of the amine to the $\mathrm{CO}_{2}$ molecules in the gas flow. This new strategy no doubt increased the utilization of template micelles occluded in the channel of SBA-15, turning waste into assets. Besides, with the similar $\mathrm{CO}_{2}$-adsorptive capacity, using the P-123 occluded SBA-15 as the host to load the same amount of tetraethylenepentamine (TEPA) could save $40 \mathrm{wt} .-\%$ of the expensive mesoporous silica, which is vital for the consumers of $\mathrm{CO}_{2}$-adsorbent. ${ }^{9}$

Similarly, we utilized their relative regular geometric structure of 
CTAB template micelles inside the channel to disperse amine and to filter the phenol in gas flow. The existence of CTAB micelles in MCM41 improves the accessibility of amine guest to the phenol molecules in the gas flow. With the same loading amounts of amine (10\%), the adsorbent based on the CTAB occluded MCM-41 sample possesses a doubled phenol adsorption capacity in comparison with common template-free MCM-41. ${ }^{10}$ The template micelles reserved in the assynthesized MCM-41 could promote the adsorption of phenol in gaseous phase, especially in the laboratorial instantaneous adsorption at $353 \mathrm{~K}$, and enables TEPA modifier to be well dispersed inside the channel of MCM-41, forming the efficient adsorbent superior to activated carbon. There were seven phenols in the mainstream smoke of cigarette, and among them quinol and catechol were the primary ones. Common MCM-41 trapped 5\% of the phenols in smoke because cigarette smoke contained thousands compounds, while activated carbon was inactive. Nonetheless, coating amine of $10 \%$ made the as-synthesized MCM-41 to reduce $18 \%$ of quinol and $15 \%$ of catechol in the smoke while other phenols components were decreased about $15 \%$ therefore the phenols level of smoke was lowered $18 \% .^{10}$

Recently, we reported two recent research progresses on this subject, using these as-synthesized mesoporous silica as new drug releasers. ${ }^{11,12}$ One was the temperature-controlled release of menthol (5-methyl-2-isopropyl-cyclohexanol) in order to check whether these micelles-occluded vessels can hold or seal volatile guests only through physical interception. ${ }^{11}$ Menthol is a natural compound to provide typical minty smell and flavour such as the flavoring agent in cigarette. Addition of menthol into cigarettes can produce the cool and refreshing taste, but its function is significantly reduced by menthol volatilization. To conquer this problem, the as-synthesized mesoporous silica MCM-41 (as-M41) adsorbed and stored as well as released menthol based on the variable flexibility of the CTAB micelles. The as-M41 composite could trap the menthol of 10 16 wt.$\%$ in $353 \mathrm{~K}-413 \mathrm{~K}$ due to the flexibility of the micelles at the relatively higher temperature. Once the temperature was lowered to ambient, the micelles exhibited inflexibility and became tetanic to block the channel so that the adsorbed menthol was sealed. After open storage of 30 days at ambient temperature, they still release the menthol at $333 \mathrm{~K}$. Once $30 \mathrm{mg}$ the as-M41 vessel, which adsorbed menthol at $373 \mathrm{~K}$ and open stored for 60 days, was put into the cigarette filter and smoked by technician, it was reported to feel the cool and refreshing of menthol when the cigarette was lighted.

Another is the new strategy of in situ loading and release of drug in mesoporous silica SBA-15, ${ }^{12}$ in which hydrophilic (Heparin) or hydrophobic drug (Ibuprofen) is encapsulated directly into vessel by using Evaporation-Induced Self Assembly (EISA) one-pot synthesis. Compared with various common loading mthods, this strategy dramatically elevated the loading amount and release proportion of drug, through which the stability of the micellar „core“ encapsulating drug in vessel would be stabilized by the mesoporous silica "shell“", and all drugs could be in situ introduced into the composites during synthesis process to avoid the extra time for drug-adsorption and the waste of drug in traditional post-loading route. Moreover, the highest temperature used in whole synthesis was $310 \mathrm{~K}$ in order to prevent deactivation of drug. Here the micelles of P123 was elected as both the structure-directing agent to construct the mesoporous vessel and the initiator to control the release of drug. In situ loading heparin, a model drug due to its wide application in prophylaxis and treatment of deep vein thrombosis and pulmonary embolism, in EISA synthesis really increased the amount of drug within the mesoporous silica vessel, reaching the highest heparin content of $167 \mathrm{mgg}^{-1}$, four times higher than that impregnated in SBA-15 $\left(29 \mathrm{mgg}^{-1}\right),{ }^{13}$ and $45 \%$ more than the highest value reported on the organic modified SBA-15 $\left(114 \mathrm{mgg}^{-1}\right){ }^{14}$ In the release stage at $45^{\text {th }}$ day, new vessel still showed an obvious release and its accumulated amount reached $70 \mathrm{mgg}^{-1}$ and the released proportion was $55.5 \%$, much higher than that of SBA-15 $\left(15.4 \mathrm{mgg}^{-1}\right.$, $53.1 \%)$. On the other hand, ibuprofen, that is widely used as a safe anti-inflammatory and analgesic drug for the relief of symptoms of inflammation, acute pain and fever, was simply immobilized into the composite through this one-pot synthesis, and the amount could achieve to $200 \mathrm{mgg}^{-1}$ that was $166 \%$ higher than that on SBA-15 by impregnation $\left(75 \mathrm{mgg}^{-1}\right)$. Moreover, all of the novel vessels had the higher release/loaded ratio (89.9 100\%) than that of SBA-15 (66\%), offering a promising candidate for release the poorly soluble drug. Through the strategy of in situ loading the drug was encapsulated inside mesoporous vessel and well mixed with template, which reduced sample preparation time to $10 \mathrm{~h}$ and utilized template micelles to adjust the release of drug. The immobilized amount of heparin or ibuprofen in vessel was greatly enhanced to 167 or $200 \mathrm{mgg}^{-1}$, giving a sustainable release longer than 30 days.

Strictly speaking, the as-synthesized mesoporous silica is the core-shell type composite where the inorganic shell forms the ordered and limited space and more importantly, the organic core also forms a magical delicate configuration, dividing further the limited space to numerous sub-nanometer gaps. Such elaborate structure is expected to provide a perfect configuration for adsorption. Lately, the as-synthesized MCM-41 (as-M41) was utilized to capture the tobacco specific nitrosamines (TSNA) that are well known as strong carcinogens, in the concentrated tobacco extract solution in order to control the pollution of TSNA in source. As expected, the as-M41 composite exhibited a high ability in trapping TSNA in the tobacco solution with thousands components, and its capability reached $0.355 \mathrm{nmolm}^{-2},{ }^{15}$ hundred times higher than that of common MCM$41\left(0.003 \mathrm{nmolm}^{-2}\right)$, providing a valuable clue to lower smokinginduced pollution. Yet, it should be pointed out that the distribution of CTAB micelles in the as-synthesized MCM-41, say, the spoke-like configuration and the numerous sub-nanometer gaps formed between these micelles and the silica walls, are very difficult to be duplicated so far. ${ }^{3}$ Therefore the regeneration and recycle used of these functional materials based on the as-synthesized mesoporous silica become a challenge facing chemists, and relevant research is underway.

\section{Acknowledgements}

Financial support from NSF of China (21273106 and 21173117), and the Analysis Centre of Nanjing University is gratefully acknowledged.

\section{Conflict of interest}

The author declares no conflict of interest.

\section{References}

1. Kresge CT, Leonowicz ME, Roth WJ, et al. Ordered Mesoporous Molecular Sieves Synthesized by a Liquid-Crystal Template Mechanism. Nature. 1992;359:710-712.

2. Zhao DY, Feng J, Huo Q, et al. Triblock Copolymer Syntheses of Mesoporous Silica with Periodic 50 to 300 Angstrom Pores. Science. 1998;279(5350):548-555. 
3. Yue MB, Sun LB, Cao Y, et al. Efficient $\mathrm{CO}_{2}$-capturer derived from the as-prepared MCM-41 modified with amine. Chemistry. 2008;14(11):3442-3445.

4. Wang YM, Wu ZY, Shi LY, et al. Rapid functionalization of mesoporous materials: directly dispersing metal oxides into as-prepared SBA-15 occluded with template. Adv Mater. 2005;17(3):323-327.

5. Wang YM, Wu ZY, Zhu JH. Surface functionalization of SBA-15 by the solvent-free method. Journal of Solid State Chemistry. 2004;177(10):3815-3823.

6. Wang YM, Wu ZY, Wang HJ, et al. Fabrication of Metal Oxides Occluded in Ordered Mesoporous Hosts via a Solid-State Grinding Route: The Influence of Host-Guest Interactions. Adv Funct Mater. 2006;16(18):2374-2386.

7. Li YY, Wan MM, Zhu JH. Cleaning carcinogenic nitrosamine with zeolites. Environmental Chemistry Letters. 2014;12(1):139-152.

8. Yue MB, Sun LB, Cao Y, et al. Promoting the $\mathrm{CO}_{2}$ adsorption in the amine containing SBA-15 by hydroxyl group. Microporous Mesoporous Mater. 2008;114(1-3):74-81.

9. Yue $\mathrm{MB}$, Chun $\mathrm{Y}$, Cao $\mathrm{Y}$, et al. Novel $\mathrm{CO}_{2}$-capturer derived from the as-prepared SBA-15 occluded by template. Adv Funct Mater. 2006;16(13):1717-1722
10. Zhou Y, Tao YF, Yang J, et al. Novel phenol capturer derived from the as-synthesized MCM-41 occluded by template micelles. J Hazard Mater. 2011;190:87-93.

11. Dong XY, Li YY, Wei F, et al. Novel menthol releaser derived from assynthesized mesoporous silica. RSC Advances. 2015;5:5494-5500.

12. Wan MM, Li YY, Yang T, et al. In situ loading of drugs into mesoporous silica SBA-15. Chem Euro J. 2016;22(18):6294-6301.

13. Wan MM, Qian WJ, Lin WG, et al. Multiply functionalization of SBA15 mesoporous silica in one-pot: Fabricating aluminum-containing plugged composite for heparin sustaining release. J Mater Chem B. 2013;1(32):3897-3905.

14. Qian WJ, Wan MM, Lin WG, et al. Fabricating a sustained release of heparin using SBA-15 mesoporous silica. J Mater Chem B. 2014;2(1):92101 .

15. Li SH, Sun XD, Wang YZ, et al. Fabricating an optimal microenvironment within mesoporous silica MCM-41 for capture of tobacco specific nitrosamines (TSNA) in solution. ACS Appl Mater Interfaces. 2017;9(32):26805-26817. 